\title{
Alta Prevalencia de Anticuerpos contra Bartonella y Babesia microti en Poblaciones Rurales y Urbanas en dos Provincias de Córdoba, Colombia
}

\author{
A high prevalence of antibodies against Bartonella and Babesia \\ microti has been found in villages and urban populations in \\ Cordoba, Colombia
}

Francisco Buelvas ${ }^{1}$, Nelson Alvis ${ }^{2}$, Indira Buelvas ${ }^{1}$, Jorge Miranda ${ }^{1}$ y Salim Mattar ${ }^{1}$

1 Universidad de Córdoba, Instituto de Investigaciones Biológicas del Trópico. Facultad de Medicina Veterinaria. Montería, Córdoba, Colombia. buelvasfrancisco@hotmail.com, jorgemire@hotmail.com, indirab65@yahoo.es, mattarsalim@hotmail.com

2 Universidad de Cartagena, Departamento de Investigaciones Económicas y Sociales. E-mail: nalvis@yahoo.com

Recibido 6 Marzo 2007/Enviado para Modificación 2 Diciembre 2007/Aceptado 17 Enero 2008

\section{RESUMEN}

Objetivo Establecer la seroprevalencia contra Bartonella henselae, Bartonella quintana y Babesia microti, en poblaciones expuestas y no expuestas a animales domésticos del sector agropecuario en el departamento de Córdoba-Colombia. Métodos Se analizaron 80 sueros de personas de una muestra no probabilística de de los municipios de Montería y Cereté del departamento de Córdoba, Colombia. La presencia de anticuerpos séricos IgG específicos anti-Bartonella henselae, Bartonella quintana y Babesia microti se llevo a cabo por la técnica de inmunofluorescencia indirecta (IFI), utilizando el kit (Focus Technologies, Cypres, Ca, USA).

Resultados Se encontró una seroprevalencía total de anticuerpos IgG anti-bartonella de $48,7 \%$ (39/80), de los cuales el $77 \%$ fueron hombres. Para Bartonella quintana la seropositividad fue de $45 \%$ (36/80), para Bartonella henselae fue de $30 \%(24 / 80)$ y en $26,2 \%$ se hallaron anticuerpos para ambas especies. Para Babesia microti la seroprevalencia fue de 30,6 \% (23/80), en la cual el $65 \%$ fueron hombres.

Conclusión La alta seroprevalencia de Bartonella y Babesia encontrada demuestra una infección subestimada en Colombia. Las autoridades sanitarias y el personal médico de la Costa Caribe de Colombia, deberían tomar decisiones con los datos obtenidos para vigilar la distribución, propagación e identificación de las poblaciones humanas en riesgo de contraer infecciones por estos microorganismos y además para orientar el diagnóstico y poder desarrollar estrategias de prevención adecuadas para estas enfermedades. 
Palabras Clave: Bartonella, Babesia microti, Colombia, Enfermedad por arañazo de gato (fuente: DeCS, BIREME).

\begin{abstract}
Objective Establishing seroprevalence against Bartonella and Babesia microti in a rural population exposed and/or non-exposed to domestic animals in Cordoba, Colombia.

Methodology Sera samples taken from 80 people from Montería and Cereté (Córdoba department) were analysed; the population sample was chosen by non-probabilistic means. Anti-Bartonella and Babesia microti were detected by indirect immunofluorescence (IFI), (Focus Technologies, Cypres, CA, USA).

Results Total anti-bartonella IgG seroprevalence was 48,7 \% (39/80), $77 \%$ being male and $23 \%$ female. Bartonella quintana seropositivity was $45 \%(36 / 80)$ and Bartonella henselae seropositivity $30 \%$ (24/80); 21 of these individuals $(26,2 \%)$ had antibodies to both bartonellas. Babesia microti seroprevelence was 30,6\% (23/80), $65 \%$ being male patients.

Conclusions High Bartonella and Babesia seroprevelence showed that infection levels had been underestimated in Colombia. Medical and sanitary authorities on the Caribbean coast of Colombia must take measures for monitoring the distribution, propagation and identification of human populations at risk of contracting infection by these micro-organisms and also orientate diagnosis and enable suitable prevention strategies to be developed for these diseases.
\end{abstract}

Key Words: Bartonella, Babesia microti, Colombia, cat scratch disease (source: $\mathrm{MeSH}, \mathrm{NLM})$.

L a enfermedad del arañazo del gato (EAG), la angiomatosis bacilar (AB) y la babesiosis no son patologías de declaración obligatoria en Colombia, no existen estudios en Colombia de seroprevalencia y de casos que demuestren la carga real de estas enfermedades en el país.

La enfermedad del arañazo del gato (EAG) es la manifestación clínica más frecuente de las infecciones causadas por Bartonella henselae, transmitida por arañazos y mordeduras de gatos (1). Bartonella quintana agente causal de la fiebre de las trincheras, bacteremias, endocarditis y de la angiomatosis bacilar es transmitido solo al hombre por piojos humanos (2). Ambas especies mantienen similitudes genéticas, usan a los mamíferos como reservorios, infectan a los mismos tipos de células (endoteliales y eritrocitos) y producen cambios vasculoproliferativos en los pacientes inmunodeprimidos Sin embargo, existen diferencias en el reservorio, $B$. quintana usa sólo al hombre y $B$. henselae utiliza al gato (2). Por otra parte, la babesiosis es considerada como una zoonosis emergente, de distribución mundial, causada por un parasito intraeritrocitico 
del género Babesia, Babesia microti, es trasmitido a los humanos principalmente por él género de garrapatas Ixodes y en menor proporción por transfusiones sanguíneas (3). La babesiosis es causada por varias especies del género Babesia que afectan diferentes animales domésticos y salvajes, así como al hombre. Las especies que más provocan afecciones en el hombre son: $B$. microti, $B$. bovis, $B$. divergens y B. bigemina (4). Se ha demostrado que Babesia bovis, divergens y bigemina afectan más a pacientes esplenectomizados, ancianos e inmunosuprimidos, mientras Babesia microti se presenta más en personas inmunocompetentes (3).

Colombia, posee características geoclimáticas para que ocurra la interacción parásito-vector-huésped, debido a la masiva infestación de vectores (garrapatas) que se encuentran distribuidos en la mayoría de las zonas geográficas del país y en el departamento de Córdoba; esta zona reúne las condiciones propicias que contribuyen al mantenimiento del ciclo biológico de muchas enfermedades transmitidas por estos artrópodos.

El objetivo de este estudio fue el de determinar la prevalencia de anticuerpos IgG anti-Bartonella henselae, Bartonella quintana y Babesia microti, en poblaciones de la provincia de Córdoba, expuestas y no expuestas a animales domésticos del sector agropecuario en el departamento de Córdoba.

\section{MATERIALES Y MÉTODOS}

Población y muestra. La población la constituyeron los habitantes de los municipios de Montería y Cereté del Departamento de Córdoba Colombia, de los cuales se tomó una muestra no probabilística de personas residentes tanto en el sector rural como urbano. Montería tiene una población urbana y rural de 272479 y 75689 respectivamente mientras que el municipio de Cereté cuenta con una población urbana de 55459 y rural de 35 049. Ochenta sujetos aceptaron participar en el estudio.

Recolección de los datos. A todas los sujetos se les aplicó una encuesta epidemiológica que indagó sobre aspectos como edad, sexo, antecedentes clínicos, síntomas y factores de riesgo asociados a la transmisión de estos microorganismos como convivencia permanente con animales domésticos, picaduras por garrapatas, mordeduras o arañazos de gatos, esplenectomía, uso de medicamentos, ocupación y viajes al exterior durante los últimos años. 
Métodos serológicos. A cada participante se le extrajo $10 \mathrm{ml}$ de sangre venosa para la detección de anticuerpos. Se estableció la presencia de anticuerpos séricos IgG específicos anti-Bartonella henselae, Bartonella quintana y Babesia microti por la técnica de inmunofluorescencia indirecta (IFI), utilizando la prueba de Focus Technologies, (Cypres, Ca, USA). La seroreactividad se observo en un microscopio de fluorescencia, mediante observación de fluorescencia verde manzana brillante sobre los bacilos fijados en el portaobjeto. La sensibilidad esperada del ensayo es de 88-95\%, en cada prueba se usó un control negativo y uno positivo. Los procedimientos fueron realizados de acuerdo a las indicaciones del fabricante. Las muestras fueron procesadas en el Laboratorio del Instituto de Investigaciones Biológicas del Trópico, Facultad de Medicina Veterinaria de la Universidad de Córdoba.

Análisis de los resultados. Los datos fueron recolectados mediante un formulario estandarizado y tabulados en una hoja electrónica de MS Excel ${ }^{\circledR}$.

Aspectos éticos. El estudio aplicó las normas para investigación de riesgo mínimo planteadas en Resolución 8430/93 del Ministerio de Salud y fue aprobado por el Comité de Ética del Instituto de Investigaciones Biológicas del Trópico. Se obtuvo consentimiento oral y/o escrito de los participantes.

\section{RESULTADOS}

De los 80 pacientes estudiados, 54 (67,5 \%) fueron hombres y 26 mujeres (32,5 \%). El rango de edad estuvo entre 15 y 85 años con una media de 41,9 años $(\mathrm{DE}=17,5)$. La procedencia de los pacientes fue de los municipios de Montería (56/80) y Cereté (24/80). En la Tabla 1, se describen las características sociodemográficas de la población estudiada y la referencia de síntomas. Se encontró una seroprevalencía total de anticuerpos IgG anti-bartonella de 48,7 \% (39 personas), $77 \%$ hombres (30 sujetos) y $23 \%$ mujeres (9 personas). Para Bartonella quintana la seropositividad fue de $45 \%$ (36/80), para Bartonella henselae fue de 30 \% (24/80) y veintiuno (26,2 \%) tuvieron anticuerpos para ambas especies.

Para Babesia microti la seroprevalencia de IgG anti-babesia fue 30,6 \% (23 individuos). La seroprevalencía encontrada en hombres fue de 65 \% (15 sujetos), mientras que la seroprevalencía en mujeres fue de $35 \%$ (8 personas).

Se observo que la exposición a animales domésticos fue del $60 \%$. (48 individuos) La exposición y la seropositividad a Bartonella spp y Babesia 
microti para las personas expuestas a animales y las personas que reportaron no tener contacto permanente con ninguno animal se muestra en la Tabla 2. Entre los individuos que no estuvieron en contacto con animales hubo menos proporción de seropositivos (Bartonella spp 41 \%) y (Babesia microti 26,1 \%). El 23 \% (19/80) de los pacientes manifestaron haber estado en contacto con por lo menos uno de los factores de riesgo. Las mordeduras o arañazos por gatos, constituyo el factor más predisponente para la transmisión de estos microorganismos ya que de 5 muestras obtenidas 2 resultaron positivas, seguido por las picaduras por pulgas o garrapatas que de cuatro muestras una fue positiva (Tabla 3).

Tabla 1. Características sociodemográficas de la población estudiada

\begin{tabular}{|c|c|c|c|}
\hline Caracteristicas & $\begin{array}{l}\text { Mujer } \\
\mathrm{n}(\%)\end{array}$ & $\begin{array}{c}\text { Hombre } \\
\mathrm{n}(\%)\end{array}$ & $\begin{array}{c}\text { Total } \\
n(\% \text { del total })\end{array}$ \\
\hline \multicolumn{4}{|l|}{ Procedencia } \\
\hline Cereté & $7(29,2)$ & $17(70,8)$ & $24(30,0)$ \\
\hline - Rural & $(0,0)$ & $8(100,0)$ & $8(10,0)$ \\
\hline - Urbana & $7(43,8)$ & $9(56,3)$ & $16(20,0)$ \\
\hline Montería & $19(33,9)$ & $37(66,1)$ & $56(70,0)$ \\
\hline - Rural & $3(16,7)$ & $15(83,3)$ & $18(22,5)$ \\
\hline - Urbana & $16(42,1)$ & $22(57,9)$ & $38(47,5)$ \\
\hline \multicolumn{4}{|l|}{ Ocupación } \\
\hline Agricultor & $3((11,5)$ & $23(88,5)$ & $26(32,5)$ \\
\hline Otros oficios & $1(5,0)$ & $19(95,0)$ & $20(25,0)$ \\
\hline Ama de casa & $14(93,3)$ & $1(6,7)$ & $15(18,8)$ \\
\hline Estudiante & $7(46,7)$ & $8(53,3)$ & $15(18,8)$ \\
\hline Profesional & $1(25,0)$ & $3(75,0)$ & $4(5,0)$ \\
\hline \multicolumn{4}{|l|}{ Sintomas } \\
\hline Ninguno & $16(25.4)$ & $47(74,6)$ & $63(78,8)$ \\
\hline Cefalea & $5(62,5)$ & $3(37,5)$ & $8(10,0)$ \\
\hline Cefalea, mareo & $2(66,7)$ & $1(33,3)$ & $3(3,7)$ \\
\hline Mareo & $1(50,0)$ & $1(50,0)$ & $2(2,4)$ \\
\hline Otros (fiebre) & $2(50,0)$ & $2(50,0)$ & $4(5,1)$ \\
\hline TOTAL & $26(32,5)$ & $54(67,5)$ & $80(100,0)$ \\
\hline
\end{tabular}

Tabla 2. Seroreactividad y exposición a animales domésticos de las personas estudiadas

\begin{tabular}{lccccc}
\hline \multicolumn{1}{c}{ Animales } & $\begin{array}{c}\text { Mujeres } \\
\mathrm{n}(\%)\end{array}$ & $\begin{array}{c}\text { Hombres } \\
\mathrm{n}(\%)\end{array}$ & $\begin{array}{c}\text { Total } \\
\mathrm{n}(\%)\end{array}$ & $\begin{array}{c}\text { Bartonella } \\
\text { spp IgG } \\
\text { positivos }\end{array}$ & $\begin{array}{c}\text { B. microti } \\
\text { IgG } \\
\text { positivos }\end{array}$ \\
\hline Ninguno & $7(21,9)$ & $25(78,1)$ & $32(40)$ & $16(41,0)$ & $6(26,1)$ \\
Bovino, gato, perro & $5(31,3)$ & $11(68,8)$ & $16(20)$ & $8(20,5)$ & $9(39,1)$ \\
Bovino, perro & $4(26,7)$ & $11(73,3)$ & $15(18,8)$ & $5(12,8)$ & $5(21,7)$ \\
Gato, perro & $10(66,7)$ & $5(33,3)$ & $15(18,8)$ & $8(20,5)$ & $3(13,0)$ \\
Bovino & 0,0 & $1(100,0)$ & $1(1,3)$ & $1(2,6)$ & $0(0,0)$ \\
Perro & 0,0 & $1(100,0)$ & $1(1,3)$ & $1(2,6)$ & $0(0,0)$ \\
\hline Total & $26(32,5)$ & $54(67,5)$ & $48(100)$ & $39(100)$ & $23(100)$ \\
\hline
\end{tabular}


Tabla 3. Prevalencia de factores de riesgo en la población estudiada

\begin{tabular}{llccc}
\hline Factor de riesgo & & $\begin{array}{c}\text { Mujer } \\
n(\%)\end{array}$ & $\begin{array}{c}\text { Hombre } \\
n(\%)\end{array}$ & $\begin{array}{c}\text { Total } \\
n=80(\%)\end{array}$ \\
\hline Paludismo & No & $25(34,7)$ & $47(65,3$ & $72(90,0)$ \\
\multirow{2}{*}{ Viajes al exterior } & $\mathrm{Si}$ & $1(12,5)$ & $7(87,5$ & $8(10,0)$ \\
& No & $25(31,6)$ & $54(68,4$ & $79(98,8)$ \\
\multirow{2}{*}{ Arañazos } & $\mathrm{Si}$ & $1(100,0)$ & $(0,0)$ & $1(1,3)$ \\
& No & $23(30,7)$ & $52(69,3$ & $75(93,8)$ \\
\multirow{2}{*}{ Picaduras } & $\mathrm{Si}$ & $3(60,0)$ & $2(40,0$ & $5(6,3)$ \\
& $\mathrm{No}$ & $22(31,9)$ & $47(68,1$ & $69(86,3)$ \\
& $\mathrm{Si}$ & $4(36,4)$ & $7(63,6$ & $11(13,8)$ \\
\hline
\end{tabular}

\section{DISCUSION}

En Colombia, la EAG y la babesiosis no son enfermedades de notificación obligatoria, por lo que su relevancia en salud pública nacional no ha sido determinada. La infección se ha descrito a nivel mundial y existen algunas encuestas serológicas que informan su prevalencia en diversas poblaciones como la reportada en Alemania 30 \% (6) y a la reportada en dueños de gatos en España, con 29 \% (7).

Estudios realizados por investigadores del Centro de Control de Enfermedades de Atlanta (CDC), EE.UU, han estimado que títulos de IgG específica anti-Bartonella henselae iguales o mayores a 64 se correlacionan con enfermedad aguda, ya que se encuentran en más del 85 \% de los casos clínicos sintomáticos de EAG y sólo en $3 \%$ de los controles sanos (1). Por ello, en ese país, se considera que la presencia de IgG anti- Bartonella henselae en título de 64 en un sujeto sin manifestaciones clínicas de EAG es indicativo de exposición previa a la bacteria y es por tanto, el nivel de corte usado para estudios de seroprevalencia.

Bartonella quintana, transmitida por piojos (Pediculus humanus) es el agente histórico de la fiebre de las trincheras que se estima que infecto a mas de 1 millón de personas durante de la primera guerra mundial y que últimamente ha sido asociado con endocarditis, fiebre y bacteriemia asintomática en pacientes inmunocompetentes e inmunocomprometidos (8). Nuestros resultados para $B$. quintana (45 \%) son altos comparados con estudios serologicos realizados en poblaciones sanas de Brasil y Grecia donde tuvieron seroprevalencías de 12,8 \% (9) y $15 \%$ (10) respectivamente.

Los resultados obtenidos por nosotros son comparables con los estudios realizados en indigentes en Tokio, Japón, donde el 57 \% (86/151) de los indigentes 
tuvieron títulos de anticuerpos positivos de 1:128. En ese mismo estudio se analizó a una población de donadores de sangre y se encontró una seroprevalencia de 51 \% (101/200) (8). Brouqui, en un hospital de Francia reportó una alta seroprevalencia en indigentes del 30 \% (11). Recientemente, Smith reportó un $10 \%$ en Los Ángeles (12).

Entre los factores de riesgos asociados a la bartonellosis están los arañazos por gatos. De los 5 sujetos que reportaron arañazos por gatos, el $40 \%(2 / 5)$ tuvieron anticuerpos IgG anti- Bartonella. Sin embargo, se ha documentado personas infectadas con Bartonella spp que solo han tenido contacto con perros (13). En nuestro estudio, de los 47 sujetos en contacto con animales, 16 solo presentaron contacto con perros, y en estos la seropositividad fue de 37,5 $\%(6 / 16)$. Aunque existió seropositividad en sujetos en contacto permanente con animales, no se encontraron diferencias significativas de seropositividad entre las personas que estuvieron en contactos con animales 46,8 \% (22/47) y las personas que no tuvieron en contacto 48,4 \% (16/33), igual como lo reportó Sander en un estudio realizado en Alemania (6). En nuestro caso esto se debe, a que la población cordobesa vive en áreas semi-urbanas por ser una zona de gran actividad agropecuaria y el contacto a los vectores es permanente. Por otra parte, la babesiosis es una enfermedad típicamente transmitida por garrapatas, La mayoría de los estudios de prevalencia de babesiosis en humanos han sido llevados a cabo en Norte América y poco se conoce acerca de la prevalencia de esta enfermedad en otras áreas. En los Estados Unidos, la gran parte de casos son debidos a Babesia microti, un parásito de pequeños roedores, particularmente el ratón de patas blancas Peromyscus leucopus, transmitido por la garrapata Ixodes scapularis. La infección se considera endémica en la región costera noreste de EEUU (14).

La seroprevalencia de Babesia encontrada en este estudio, 30,6 \% es alta comparada por la encontrada por Foppa en Suiza, seroprevalencia del 1,5 \% (16), Walory en Polonia quien reportó prevalencias de 1,4 \% de B. microti (15), Bronsdon en Europa seroprevalencias de $4 \%$ (17) y de 11, $5 \%$ seroprevalencia reportada por Hunfeld de pacientes expuestos a picaduras por garrapatas en Alemania (18).

En Colombia solo se han realizados estudios tendientes al diagnostico inmunológico y microscópico de Babesia bigemina y Babesia bovis, Ríos (19). Este es el primer reporte serológico de B. microti en el la región caribe. Tal vez una de las razones por la cual en el país no se tiene evidencia clínica reportada 
de la enfermedad por este microorganismo se debe a que en la mayoría de los casos de B. microti usualmente se resuelven sin tratamiento (3).

La sintomatología reportada por los sujetos incluidos en el estudio mostró que 13,7 \% (11/80) de los sujetos reportaron cefalea, otros síntomas fueron: fiebre $2,5 \%$ (2/80) y mareos 2,5 \% (2/80). Ningún paciente describió linfadenopatia regional que es signo clínico más indicativo de la EAG. Esta presentación clínica observada en este estudio no es la característica de los pacientes con enfermedad por arañazo de gato, angiomatosis bacilar, fiebre de las trincheras como lo reportan otros autores $(20)$ y por babesiosis $(14,15)$.

En consideración al diagnóstico de EAG y babesiosis es necesario mejorar el conocimiento de estas infecciones en Colombia. Es sabido que el diagnostico, de estas patologías es difícil ya que Bartonella es un género de difícil y lento crecimiento en cultivos y el éxito del aislamiento es posible si la muestra es recogida en etapas tempranas de la enfermedad (13). El diagnostico clínico y de laboratorio de Babesiosis es también difícil, por lo que las técnicas de inmunofluorescencia se convierten en una herramienta útil para el diagnostico temprano de estas patologías en el laboratorio clínico.

Aunque la muestra analizada no es representativa de la población, con base en los resultados obtenidos en este estudio preliminar, seria importante ampliar este tipo de estudios a otras poblaciones humanas y animales en estas provincias de Córdoba y el caribe Colombiano. La alta seroprevalencia encontrada para cada uno de los microorganismos estudiados Bartonella quintana $45 \%$, Bartonella henselae 30 \% y Babesia microti 30,6 \%, demuestra por primera vez en Colombia un importante problema de salud pública subestimado •

\section{REFERENCIAS}

1. Ferres M, Abarca K, Prado P, Montecinos L, Navarrete M, Vial PA. Prevalencia de anticuerpos contra Bartonella hensalae en niños, en adolescentes y en una población de riesgo ocupacional en Chile. Rev Med Chile 2006; 134: 863-867.

2. Alsmark CM, Frank AC, Karlberg EO, Legault BA, Ardell DH, Canback B, et al. The louse-borne human pathogen Bartonella Quintana is a genomic derivative of the zoonotic agent Bartonella henselae. Proc Natl Acad USA 2004; 101: 97169721.

3. Homer MJ, Aguilar-Delfin I, Telford III SR, Krause PJ, Persing DH. Babesiosis. Clin Microbiol Rev 2000; 13: 451-469. 
4. Suárez M, Alonso M, Peláez R, Sánchez B, Bravo J, Sánchez A. Pesquisaje de Babesia en trabajadores agropecuarios y donantes en la provincia de Ciego de Ávila. Rev Cubana Med Trop 1997; 49: 130-135.

5. World medical association declaration of Helsinki. Ethical principles for medical research involving human subjects, WMAgeneral asambly, tokio, octubre 2004. [Internet]. Disponible en: http://www.wma.net/e/policy/b3.htm. Consultado: abril de 2005.

6. Sander A, Possel M, Oberle K, Bredt W. Seroprevalence of antibodies to Bartonella hensalae in patients with cat scratch disease and healthy controls: evaluation and comparison of two commercial serological test. Clin Diagn Lab Immunol 1998; 5: 486-490.

7. Blanco JR, Oteo JA, Martínez V, Ramalle E, García A, Ibarra V. Seroepidemiology of Bartonella henselae infection in a risk group. Rev Clin Esp 1998; 198: 805-809.

8. Seki N, Sasaki T, Sawabe K, Sasaki T, Matsuoka M, Arakawa Y, et al. Epidemiological studies on Bartonella quintana infections among homeless people in Tokyo, Japan. Jpn J Infect Dis 2006; 59: 31-35.

9. Costa PSG, Brigatte ME, Greco BD. Antibodies to Rickettsia rickettsii, Rickettsia typhi, Coxiella burnetii, Bartonella henselae, Bartonella quintana, and Ehrlichia chaffeensis among healthy population in Minas Gerais, Brazil. Mem Inst Oswaldo Cruz 2005; 100: 853-859.

10. Tea A, Alexiou-Daniel S, Diza AE, Antonadis A. Ocurrence of Bartonella henselae and Bartonella quintana a healthy greek population. Am J Trop Med Hyg 2003; 68:554-556.

11. Brouqui P, Lascola B, Roux V, Raoult D. Chronic Bartonella quintana bacteremia in homeless patients. N Engl J Med 1999; 340: 184-189.

12. Smith HM, Reporter R, Rood MP, Linscott AJ, Mascola LM, Hogrefe W, et al. Prevalence study of antibody to ratborne pathogens and other agents among patients using a free clinic in downtown Los Angeles. J Infect Dis 2002; 186: 1673-1676.

13. Huarcaya E, Maguina C, Merello J, Cok J, Birtles R, Infante B, et al. A prospective study of Cat-Scratch Disease in Lima-Peru. Rev Inst Med Trop Sao Paulo 2002; 44:325-330.

14. Herwaldt BL, Cacciò S, Gherlinzoni F, Aspöck H, Slemenda SB, Piccaluga P, Martinelli $G$ et al. Molecular characterization of a non-Babesia divergens organism causing zoonotic babesiosis in Europe. Emerg Infect Dis 2003; 9: 942-948.

15. Walory J, Bukowska B, Grzesiowski P, Czarnecka I, Paluchowska E, Zabielski S, et al. Prevalence of antibodies against Anaplasma phagocytophilum, Babesia microti, Borrelia burgdorferi in adults in North-Eastern Poland. Pol Merkur Lekarski 2005; 19: 754-757.

16. Foppa IM, Krause PJ, Spielman A, Goethert H, Gern L, Brand B, et al. Entomologic and serologic evidence of zoonotic transmission of Babesia microti, eastern Swizerland. Emerg Infect Dis. 2002; 8: 722-726. 
17. Bronsdon MA, Homer MJ, Magera JM, Harrison C, Andrews RG, Bielitzki JT, et al. Detection of enzootic babesiosis in baboons (Papio cynocephalus) and phylogenetic evidence supporting synonymy of the genera Entopolypoides and Babesia. J. Clin. Microbiol 1999; 37: 1548-1553.

18. Hunfeld KP, Lambert A, Kampen H, Albert S, Epe C, Brade V, et al. Seroprevalence of Babesia Infections in Humans Exposed to Ticks in Midwestern Germany. J. Clin. Microbiol 2002; 40: 243-2436.

19. Rios L, Alvarez G, Blair S. Serological and parasitological study and report of the first case of human babesiosis in Colombia. Rev Soc Bras Med Trop 2003; 36: 493498.

20. Valdesoiro L, Pineda V, Martin C, Sanfeliu I, Cabezas RM, Sanchez M. Abscesos esplénicos y enfermedad por arañazos de gato. An Esp Pediatr 2001; 55: 365368. 